
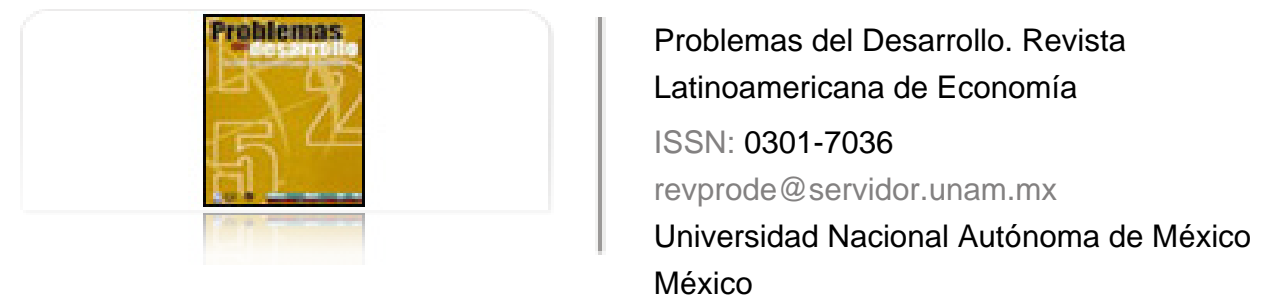

Durán E., Jorge A.; Carrillo H., Mario M.

La localización y la interrelación de la industria en regiones en desarrollo. El caso de Ciudad del

Carmen, Campeche

Problemas del Desarrollo. Revista Latinoamericana de Economía, vol. 36, núm. 140, 2005, pp. 143-

164

Universidad Nacional Autónoma de México

Distrito Federal, México

Disponible en: http://www.redalyc.org/articulo.oa?id=11820092007

- Cómo citar el artículo

- Número completo

- Más información del artículo

Página de la revista en redalyc.org

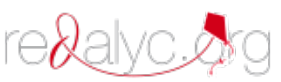

Sistema de Información Científica

Red de Revistas Científicas de América Latina, el Caribe, España y Portugal

Proyecto académico sin fines de lucro, desarrollado bajo la iniciativa de acceso abierto 


\title{
LA LOCALIZACIÓN Y LA INTERRELACIÓN DE LA INDUSTRIA EN regiones en desarrollo. El caso de Ciudad del Carmen, CAMPECHE*
}

\author{
Jorge A. Durán E.** \\ Mario M. Carrillo H.***
}

Fecha de recepción: 31 de agosto de 2004. Fecha de aceptación: 1 de marzo de 2005.

\section{Resumen}

En este trabajo se presentan los resultados de un estudio basado en una encuesta levantada en 2002 a 58 empresas industriales de Ciudad del Carmen, Campeche, clasificadas como locales (individuales y matrices) versus foráneas (filiales y de grupo), tanto nacionales como multinacionales. Con ello se buscaba identificar las actividades económicas con los mayores grados de impacto en la región y los factores más influyentes en su decisión de localización, para promover la instalación de empresas nuevas. Ello, como mecanismo de compensación de las afectaciones económicas de un proyecto de explotación de gas, iniciado a mediados de ese mismo año, el cual implicaba la construcción de plataformas marinas. En general, se encontraron niveles bajos de integración regional, sobre todo entre empresas foráneas, mientras que el factor de localización más importante para todas estuvo centrado en las posibilidades que les da el mercado para sus ventas.

Palabras clave: localización industrial, interrelación económica, impactos regionales, regiones periféricas, empresas industriales.

* Se agradece la autorización del ingeniero Javier Hinojosa Puebla, subdirector de la Región Marina Noreste (RM NE) PEM EX, para la publicación de este artículo.

* Profesor titular en la Escuela de Negocios de la Universidad de las Américas-Puebla. Correo electrónico: jduran@mail.udlap.mx. Se agradece al licenciado Antonio M artínez Tapia su valiosa participación en la elaboración del material de este trabajo.

** Profesor-investigador de El Colegio de Tlaxcala. Profesor titular de economía de la Universidad de las Américas- Puebla. Correo electrónico: mmch@ prodigy.net.mx 


\begin{abstract}
In this paper, the results are presented of a study based on a survey carried out among 58 industrial companies in Ciudad del Carmen, Campeche in 2002, classified as local (individual and matrices) vs. foreign (subsidiaries of groups), and both national and multinational. On this basis, it was sought to identify the economic activities with major degrees of impact in the region and the most influential factors in their location decision, to promote the installation of new companies. This is used as a mechanism offsetting the economic effects of a project to exploit gas, started in the middle of that year, which implied the construction of marine platforms. In general, low levels were found for regional integration, above all among foreign companies, while the most important location factor for all companies centered on the possibilities of finding a market for their sales.
\end{abstract}

Key terms: industrial location, economic interrelation, regional impacts, peripheral regions, industrial companies.

\title{
Résumé
}

Dans ce travail, on présente les résultats d'une étude basée sur une enquête menée en 2002 avec 58 entreprises industrielles de Ciudad del Carmen, Campeche, classifiées comme locales (individuelles et maisons mères) versus étrangères (filiales et de groupe), aussi bien nationales que multinationales. Le but étant d'identifier les activités économiques ayant le plus haut degré d'impact sur la région et les facteurs d'influence pour leur décision de localisation, pour promouvoir l'installation de nouvelles entreprises. Tout ceci, comme mécanisme de compensation des affectations économiques d'un projet d'exploitation de gaz, mis en place à partir de cette même année, lequel impliquait la construction de plates-formes marines. On a trouvé en général des niveaux bas d'intégration régionale, surtout parmi les entreprises étrangères, tandis que le facteur de localisation le plus important pour toutes était centré sur les possibilités que leur offrait le marché pour leurs ventes.

Mots clés: localisation industrielle, interrelation économique, impacts régionaux, régions périphériques, entreprises industrielles.

\section{Resumo}

Neste trabalho apresentam-se os resultados dum estudo baseado numa pesquisa levantada em 2002 a 58 empresas industriais de Ciudad del Carmen, Campeche, classificadas como locais (individuais e martizes) versus forâneas (filiais e de grupo), tanto nacionais quanto multinacionais. Mediante isto procurava-se identificar as atividades económicas com os maiores graus de impacto na região e os fatores de maior influência na decisão da sua localização, para promover! a instalação de empresas novas. Isto, como mecanismo de compensação das afectações económicas dum projeto de explotação de gás que começou aproximadamente à metade daquele ano, o que implicava a construção de plataformas marinas. Geralmente, acharam-se baixos niveis de integração regional, principalmente entre empresas forâneas, entanto que o fator de localização de maior importância para todas esteve centrado nas possibilidades que o mercado lhes oferece para suas vendas.

Palavras chave: localização industrial, interrelação económica, impactos regionais, regiões periféricas, empresas industriais.

\section{Desaarrollo}




\section{Introducción}

in duda, las perspectivas de crecimiento económico de una región dependen de su
capacidad para promover la localización de empresas generadoras de actividad y
empleos, independientemente de las ramas en las cuales se desempeñen. Sin embargo, dichas perspectivas de crecimiento serán mayores en la medida en que las compañías que allí se establezcan estén integradas a la región, es decir, que las mayores interrelaciones económicas enfoquen hacia los sectores o actividades que más impactan en el territorio.

En este trabajo se presentan los resultados de un estudio, realizado con base en una encuesta levantada en 2002, que tuvo dos objetivos: primero, identificar las actividades económicas con los mayores grados de integración regional, entendidos como los de mayores coeficientes técnicos intrarregionales en una matriz insumo-producto (Isard, 1971) y, segundo, identificar los factores de mayor influencia en la decisión de localización de las empresas de la región, para promover la instalación de nuevas plantas en las ramas seleccionadas, por su impacto regional, en Ciudad del Carmen, Campeche. ${ }^{1}$

El trabajo está dividido en cinco secciones, incluida la introducción. En la segunda se presenta el marco de referencia de la localización y la interrelación económicas. También se revisan los conceptos de análisis de las interrelaciones de la actividad económica, incluidos los efectos multiplicadores y de derrama económica entre las regiones. En la tercera se comenta el método utilizado en el estudio. En la cuarta, se presentan los resultados de la aplicación de la encuesta y, en la última sección, las conclusiones del estudio.

\section{Revisión de la literatura}

\section{Importancia de los factores de localización}

Desde hace muchos años se ha estudiado la importancia de los factores de localización en la atracción y creación de empresas en ciertas regiones. Este tema fue desarrollado teóricamente por economistas y geógrafos, poniendo énfasis en el papel determinante de los

1 De hecho, se trataba de compen sar las posibles afectaciones económicas de un proyecto de explotación de gas en la península de Atasta y en Ciudad del Carmen, Campeche. El proyecto en cuestión es Campeche Oriente Terciario, Cuenca M acuspana M arina (Akalán), Proyecto Estratégico de Gas de PEM EX, Cuya aplicación se inició a mediados de 2002, y consiste en la construcción de plataformas marinas frente a la península de Atasta y de Ciudad del Carmen. Cabe aclarar que en este trabajo no se abordan las afectaciones económicas mencionadas ni la selección de la cartera de los proyectos resultantes, sin embargo, sus resultados establecieron un fuerte sustento para la selección de la cartera de proyectos.

\section{$\underline{\text { DeSarerrollo }}$}


costos de transporte en la ubicación de las compañías, costos vinculados con la distancia entre ella y sus proveedores de insumos, y también con relación al mercado (Carrillo, 2002).

Sin embargo, en la práctica, las investigaciones y experiencias pusieron en evidencia la importancia de otros factores — además del transporte— que poco a poco se fueron considerando relevantes en la atracción de las empresas hacia las regiones, tales como la disponibilidad de mano de obra, de energía y su costo, la infraestructura, terrenos, ayudas gubernamentales, interrelaciones empresariales, clima y calidad de vida, entre otros (Chapman y Walter, 1987).

Una derivación de estos avances se concentró en definir las características de las economías de aglomeración, entendidas como aquellos ahorros en los costos para una empresa debido a la escala de una industria en una conurbación o región particular, y la habilidad resultante de la empresa para compartir algunos de sus costos y gastos con otras (ibid.). ${ }^{2}$

Diversas opiniones se han vertido en cuanto al grado en que estas aglomeraciones sustentan y promueven mayor especialización industrial, como lo planteó originalmente Marshall (1920) al referirse a los distritos industriales. Este autor argumentó que, al agruparse de este modo, los productores lo lograrían con mayor facilidad en diferentes etapas complementarias del proceso de producción, y conseguirían atraer proveedores de insumos especializados, así como compradores para sus productos. Además de que se crearían grupos de trabajadores con esas características y se difundirían con rapidez las ideas y prácticas novedosas. ${ }^{3}$

Al referirse a la formación de pools de trabajadores que se conforman en las regiones y se convierten en atractivo para empresas de diversos sectores industriales, Krugman (1991) postula que las aglomeraciones no reflejan necesariamente especialización sectorial.

Al reinterpretar el concepto de Marshall en una obra reciente, Peneder (2001:142) concluye que "no predice una especialización completa de las localizaciones industriales. En su lugar, es típica una composición mixta de productos a lo largo de un amplio rango de actividades en combinación con un fuerte enfoque en algunas industrias".

Actualmente, buena parte de la literatura acerca del tema presta atención al papel que desempeñan el compartimiento de información y los conocimientos especializados de la

2 Algunas de las principales ventajas que tiene la ubicación en una ciudad grande es estar cerca de la vivienda de los trabajadores, de servicios especializados como almacenadoras, arrendadoras, empacadoras, comercializadoras, transportistas, despachos de investigación demercados, de publicidad, consultores, servicios de reparación y servicios de maquinaria, banca especializada, instituciones financieras y de seguros, oficinas de gobierno, universidades, politécnicos, colegios técnicos, centro de exposiciones, y mano de obra especializada. La escala, según el nivel de actividad económica, población e infraestructura de las ciudades, crea economías externas con un efecto dinámico acumulativo que permite fomentar, organizar y distribuir la producción (Richardson, 1977).

3 Numerosos análisis de clusters contemporáneos confirman la importancia de estas economías externas en el nivel local.

\section{DeSarrollo}


fuerza laboral en el atractivo de las regiones, que se convierten en centros donde los procesos de innovación y fortalecimiento del espíritu empresarial tienden a potenciarse (Bennett et al., 2001; Roper et al., 2000; Kloosterman, 2001; Peneder, 2001).

Muy vinculado con lo anterior, se menciona cómo los factores han contribuido a reforzar los procesos de concentración industrial de ciertas regiones, ya sea al interior de diversos países o bien en la conformación de un mosaico mundial de suprarregiones o clusters industriales (Scott, 1998; Porter, 2000).

Por otra parte, el papel del transporte en la localización industrial se ha recontextualizado, al vincularse de manera importante con las características de procesos de producción actuales, que están evolucionando hacia otros de manufactura flexible e intensivos en información, a partir de procesos fordistas caracterizados por grandes complejos industriales integrados verticalmente, que privilegiaban la estandarización de productos y servicios en la búsqueda de economías de escala. La siguiente cita resume esta perspectiva:

debido a que los sistemas postfordistas y de producción flexible están fuertemente externalizados y son intensivos en transacciones, las aglomeraciones regionales parecen otra vez estar reemergiendo, en contraste con el constante resquebrajamiento de muchas regiones industriales que estaba ocurriendo conforme el fordismo alcanzaba su clímax. Las regiones una vez más emergieron como el foco de producción y repositorio de know-how especializado y de capacidades tecnológicas, aun cuando la globalización de relaciones económicas procede a un paso acelerado (Scott, 1998:100).

En México, algunos estudios han destacado el papel de diversos factores de localización en el desarrollo de las regiones. Así, Garza (1999) muestra los más importantes en las empresas manufactureras en México a fines de los ochenta, tanto en ciudades como en parques industriales y analiza los factores de localización de insumos, mano de obra, transporte, mercado e infraestructura. De hecho, hace énfasis en la relación entre la infraestructura de servicios públicos y privados, y en la posibilidad de generar economías externas o de aglomeración y concluye que:

la infraestructura del parque y de la ciudad donde éste se encuentra representa el factor central en la decisión locacional de la planta. Esto es el resultado del carácter más flexible de los otros determinantes y del elevado grado de ubicuidad de las empresas por la revolución en los medios de transporte y el cada vez menor uso de materias primas agrícolas. La mano de obra, los insumos y el capital mismo se trasladan con relativa facilidad en el espacio, pero la infraestructura es fija y tiende históricamente a acumularse en pocas ciudades y regiones (p. 307).

Para resaltar la importancia de las economías externas o de aglomeración, Hernández Laos (1977) explica que la eficiencia interna de las empresas industriales en México contribuye con $44.5 \%$ de la variación explicada de la eficiencia total y los factores externos, con $55.5 \%$.

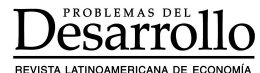


Por otra parte, otros investigadores resaltan los recientes desarrollos regionales promovidos con la participación más local de diversos agentes económicos (Graizbord,1993; Ruiz, 2000; Meyer-Stamer, 2000a y 2000b). En esta dirección, Ruiz (2000), describe tres etapas en la transformación del desarrollo económico regional en México, según los cuales, en los años sesenta el desarrollo económico local era, principalmente, una cuestión del gobierno, enfocado a la atracción de inversión fuera de la región y al desarrollo de la infraestructura dura; a esto se le llamó primera ola del desarrollo económico. La segunda surgió en los años ochenta y noventa, al buscar la consolidación y expansión de empresas locales. En esta nueva etapa fue una práctica común el apoyo a las empresas locales mediante subsidios - exención de impuestos, entrega de terrenos en parques industriales-. La tercera etapa es la que el mundo vive hoy, y consiste, básicamente, en el fortalecimiento del marco de desarrollo empresarial; la formación de socios y los enfoques estratégicos son guías para un desarrollo integral. Aunado a esto, Ruiz (2000) comenta la aplicación de ciertos programas descentralizados del gobierno federal para la promoción industrial y el apoyo a la formación del capital humano local.

\section{Clasificación de los factores de localización}

Aparte de las economías que la urbanización crea, estudios recientes han buscado agrupar los factores de localización industrial que propician los clusters industriales de acuerdo con dos tipos principales (Ellison y Glaeser, 1997):

1) Específicos de la industria. Externos a los establecimientos, pero internos al cluster industrial regional. Los beneficios que se derivan de este tipo de aglomeración son: a) mayor especialización de proveedores de insumos intermedios, servicios empresariales y mercados financieros; $b$ ) grupos más numerosos de trabajadores capacitados y entrenados, y reducción de costos de reclutamiento de trabajadores con ciertas habilidades; $c$ ) redes y comunicaciones entre empresas para aprovechar complementariedades, explotar nuevos mercados, integrar actividades y adoptar innovaciones, y $d$ ) escala para el suministro de bienes y servicios públicos a la medida de las necesidades de las industrias específicas.

2) Naturales. En su mayoría, se desprenden de atributos naturales con los cuales ha sido dotada una región. Los beneficios que se derivan de este tipo de aglomeración son: a) ventajas de costo en transacciones derivadas de distancias: localizaciones de minimización de costos de transporte, y $b$ ) capital, mano de obra, tipo de terrenos y clima.

Aunque desde una perspectiva más amplia, varios de los factores que se agrupan como naturales (distancia, capital y mano de obra) pueden entenderse como resultado de transformaciones históricas, su inclusión se sustenta en la diferenciación conceptual que hacen

\section{$\underline{\text { DeSarrofrollo }}$}


los autores entre el fenómeno urbano y el de los clusters. Al respecto, Ellison y Glaeser (1997) consideran difícil distinguir la importancia de ambos tipos de economías de aglomeración en los procesos de concentración; sin embargo, sugieren que algunos de los casos más extremos de concentración se deben, probablemente, a ventajas naturales. También advierten gran variación entre industrias.

La gran mayoría de los estudios relacionados con economías de aglomeración se han enfocado al papel de estos factores en la conformación de regiones metropolitanas en el nivel mundial y en escenarios de competencia global (Scott, 1998; Porter, 2000; Peneder, 2001). Sin embargo, dadas las características de la región a la cual se enfoca este trabajo, es conveniente considerar los enfoques de los estudios dirigidos a explicar la importancia de los factores de localización industrial en áreas no metropolitanas.

Al respecto, a pesar del escaso desarrollo socioeconómico en regiones periféricas, la elevada disponibilidad de mano de obra, motivada principalmente por desempleo, subempleo y costo reducido, a menudo llegan a ser características importantes de la mano de obra, sobre todo en países en desarrollo.

Los estudios enfatizan la manera en que las características de la estructura de la industria en este tipo de regiones pueden ser causas importantes de los procesos de aglomeración; por ejemplo, tamaño de la empresa, propiedad, multiplantas o multiproductos, integración vertical y relaciones con proveedores de insumos y de servicios, entre otros.

A continuación se consideran algunas características de concentración espacial en áreas no metropolitanas.

\section{a) Características industriales}

- Integración vertical. Pueden crear menos incentivos de aglomeración para reducir costos de transacciones y mejorar flujos de información (Enright, 1993, 1994; Holmes, 1995).

- Empresas con operaciones multiplantas. Plantas no filiales se benefician más de economías externas que otras que no lo son; como resultado, la concentración de establecimientos es más probable para plantas no afiliadas. Las filiales tienen vínculos locales de compraventa más débiles que las de propiedad local. También mantienen una proporción menor de empleados en las categorías profesionales y ejecutivas que las de pertenencia local (Henderson, 1997; Enright, 1993).

- Tamaño de la planta. Esta característica puede o no favorecer la concentración. Puede aglomerar establecimientos si las empresas grandes contribuyen a la creación de nuevas, si son atraídos los proveedores de insumos y servicios especializados, o si éstos propician el desarrollo de grupos de trabajadores con habilidades industriales específicas. Pero las grandes empresas también pueden contribuir al aumento de precios de insumos locales. Si así fuera, otros establecimientos podrían evadir localizaciones con grandes plantas y provocan la dispersión espacial. También, las grandes empresas pueden asociarse con relativamente más compras no locales (Scott, 1986; Smith y Barkley, 1991).

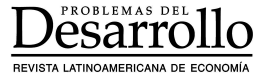


- Diversidad de productos. La diferenciación de productos entre plantas puede motivar aglomeración espacial al facilitar redes e intercambio de información entre las empresas y sus clientes. El impacto de la diferenciación al interior de la industria es menos claro, sobre todo si empresas tienen pocos vínculos o tienen requerimientos locacionales marcadamente diferentes (Kim, Barkley y Henry, 2000).

b) Características de la mano de obra

- La disponibilidad, el costo y la calidad de la mano de obra son consistentemente citados como determinantes de la localización de manufacturas. (Herzog y Schlottman, 1991; Krugman, 1991; Henderson, 1986). Al respecto, a pesar del subdesarrollo socioeconómico en regiones periféricas, los aspectos de disponibilidad abundante y costo reducido, a menudo llegan a ser características importantes de la mano de obra.

\section{c) Características del mercado}

- Estas localizaciones proveen ventajas naturales en términos de proximidad a mercados de insumos y productos. Las plantas manufactureras caracterizadas por desintegración vertical, producción en lotes pequeños o sistemas flexibles de producción son especialmente dependientes en soporte técnico y profesional externos (Malecki, 1991; Barkley y Hinschberger, 1992). Tanto la demanda intraindustrial como los vínculos de suministro, si son suficientemente fuertes, crean retroalimentaciones positivas y motivan mayor expansión de la actividad industrial en una localización (Krugman, 1991; Venables, 1994).

En un estudio reciente, Kim, Barkley y Henry (2000) utilizan un modelo de regresión múltiple para analizar las características de concentración industrial en áreas no metropolitanas de Estados Unidos en los noventa. Concluyen que los clusters espaciales de empresas están relacionados positivamente con el tamaño promedio de los establecimientos, con su dependencia en insumos de recursos naturales, intensidad de mano de obra, participación en los costos de empleados técnicos y profesionales, y el costo de trabajadores con bajas habilidades. Estas aglomeraciones se relacionan negativamente con la estructura de multiplantas, la utilización de empleados con habilidades medias (operadores) y la dependencia en mercados de productos e insumos locales.

Otros estudios han examinado las características de las regiones como fuente de progreso económico en países en desarrollo, considerando en el análisis la eficiencia lograda mediante la aglomeración de empresas de un mismo sector y de economías de urbanización. En un buen número de casos, el sustento principal de la expansión regional ha coincidido con el establecimiento de complejos de empresas pequeñas, con uso intensivo de

\section{$\underline{\text { DeSarrofrollo }}$}


mano de obra y basadas puramente en habilidades locales (Scott, 1998). Las empresas filiales de multinacionales y la subcontratación de actividades hacia dentro han sido fuente significativa de aglomeración y vehículo importante mediante el cual estas regiones se han incorporado a cadenas globales de bienes (Bellak y Cantwell, 1998; Christerson y LeverTracy, 1997; Gereffi, 1995; Henderson, 1997).

Aunque es escasa la información, se han reportado algunas experiencias de procesos de industrialización en áreas petroleras periféricas de países desarrollados. Cumbers (2000a) se interesó en examinar si la actividad de empresas filiales de compañías multinacionales en la región Grampian de Escocia, específicamente en Aberdeen, en los años ochenta, fue capaz de soportar un desarrollo de empresas regionales. El autor concluye que aunque sí se registró durante los noventa un aumento modesto en las empresas regionales manufactureras y de servicios vinculadas con la industria petrolera, su nivel de sofisticación técnica sigue siendo baja, dada la importancia estratégica del mar del Norte en el desarrollo de nuevas tecnologías, pero se apreció un proceso de mayor integración regional en el mercado laboral, soportado por programas de educación superior y de capacitación y adiestramiento en dos universidades de la región.

En otro análisis realizado por Cumbers (2000b) en la localidad de Stavenger, Noruega, la intervención del Estado nacional fue un elemento catalítico para lograr mayor integración de las firmas locales con la industria petrolera. Sin embargo, las acciones del gobierno han tenido que enfrentar fuertes intereses de un pequeño, pero muy influyente, número de actores multinacionales.

Basados en la revisión de esta literatura, decidimos elaborar un cuestionario que permitiera distinguir los factores de atracción en el establecimiento de empresas en el municipio de Carmen, Campeche y sus características de estructura industrial.

\section{Análisis del efecto multiplicador}

Una parte importante del estudio es el análisis del efecto multiplicador interregional o de derrama económica de las actividades industriales en el municipio de Carmen. De particular importancia para este análisis resultaría la presencia en la zona de industrias y empresas propulsoras cuyas principales características fueran: $a$ ) dinámicas, con un nivel avanzado de tecnología; $b$ ) con alta elasticidad de ingreso y de demanda para sus productos que usualmente sean vendidos en el nivel nacional; $c$ ) con fuertes vínculos interindustriales, hacia delante y atrás; y $d$ ) relativamente grandes, que generaran un crecimiento significativo, con habilidad para innovar y pertenecientes a industrias con crecimiento rápido. ${ }^{4}$

4 La manera tradicional de analizar el efecto multiplicador de las industrias es mediante las tablas de insumo-producto de Leontieff (1966), un conjunto de cuentas de doble entrada, usualmente en términos monetarios, preparadas para una economía. Pone atención explícita a las interrelaciones con diferentes sectores de la economía, concentrándose en particular en lasrelacionesinterindustriales.

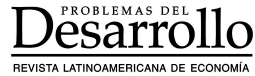




\section{Método}

Debido a la falta de información estadística con el detalle descrito y a las dificultades para preparar este tipo de tablas, decidimos elaborar un cuestionario que pudiera recoger las interrelaciones de las empresas de la región con las de otras zonas, tanto hacia adelante (hacia el mercado) como atrás (hacia las fuentes de materias primas, partes, suministros y fuerza laboral). Este cuestionario, que consta de tres partes, fue aplicado a una muestra representativa de 58 empresas del municipio de Carmen.

La primera parte consulta aspectos generales de las empresas. Posteriormente, hay preguntas que se refieren a las relaciones que se establecen con otras empresas por medio del mercado. La última parte consta de preguntas orientadas a investigar los factores que impulsaron la decisión de empresarios para instalarse en Ciudad del Carmen. ${ }^{5}$

Se contactó al director general de cada empresa, pues era de esperar que fueran ellos los actores clave al momento en que la compañía decidió establecerse en este municipio.

\section{Resultados}

Características generales de las empresas

Una consideración básica del estudio fue el interés por encontrar las diferencias entre los vínculos socioeconómicos que establecen las empresas locales (individuales y matrices) con el municipio de Carmen, en comparación con las establecidas por foráneas (filiales y de grupo). Los resultados de las diferentes preguntas del cuestionario están basados en esa consideración.

La primera pregunta solicita el nombre completo de la empresa y la segunda indaga acerca del giro. La tercera pretende determinar el tipo de compañíaa en cuanto a su propiedad. De acuerdo con estos datos, $37.21 \%$ de las empresas entrevistadas son de tipo

En estetrabajo se tratan de identificar las industrias que pueden tener mayores efectos multiplicadores dentro de la región. Una exposición amplia de las tablas, así como de sus usos y de los problemasque pueden representar su construcción y actualización oportuna, sobre todo en el nivel regional, se encuentran en Glasson (1980), Boomsma y Oosterhaven (1992), Hewings y Jonem (1986), Buetre y Ahmadi-Esfahani (2002).

5 La primera parte incluye diez preguntas que detectan nombre, giro, tipo, volumen de ventas, número de empleados, antigüedad, principales productos o servicios einsumos. Luego, tres preguntas se refieren a las relaciones interempresariales e interregionales de compra de insumos, venta de productos o servicios y procedencia de la mano de obra. Aquí se distinguieron los siguientes lugares: municipio de Carmen, otros del estado de Campeche, la ciudad de M éxico, el resto del territorio, Estados Unidos y Canadá, entre otros países. De esta manera, la información proporcionada por estas tres preguntas sustituye, en cierta medida, la información que proporcionaría una matriz de insumo-producto interregional. La última parte se compone de 62 preguntas o factores de localización, que se pueden resumir en 17 grupos de factores. Las respuestas a la importancia que dan las empresas a estos factores de localización se describen mediante una escala de Likert, que va desde $1=\sin$ importancia, hasta $5=$ muy importante.

\section{DeSRarrollo}


individual; le siguen las de tipo filial (20.93\%), las de matriz (30.23\%) y las de grupo $(11.63 \%)$.

La cuarta pregunta consulta el volumen de ventas del último año. Los resultados muestran que $37.21 \%$ de las empresas visitadas tuvo ingresos por más de 20 millones de pesos; los siguientes dos porcentajes más significativos son $16.28 \%$ y $13.95 \%$, con ingresos de entre 1 y 3 millones de pesos y de entre 5 y 8 millones de pesos, respectivamente (Cuadro 1).

Los datos muestran una estructura diferente de las ventas entre las empresas individuales y matrices, y las filiales y de grupo; el conjunto de las primeras cubre un espectro mayor de niveles de venta que las segundas e incluye un mayor número de compañías con ventas mayores de 15 millones de pesos.

La quinta pregunta del cuestionario consulta el número de empleados de las empresas entrevistadas. El Cuadro 1 muestra que la mayoría (58.15\%) tiene al menos 30 empleados, aunque algunas (13.95\%) rebasan los 100, destacando dos empresas que tienen 1000 y 1200 trabajadores.

De ahí se desprende que el grupo de empresas individuales y matrices incluye a las más grandes de la muestra en términos del número de empleados.

La sexta pregunta del cuestionario investiga acerca de la categoría del personal: 13.70\% del personal ocupado en las empresas es directivo, $14.25 \%$ desempeña puestos técnicos o de supervisión, 20.75\% labora como obrero calificado, $7.90 \%$ como obrero no calificado, $27.40 \%$ es personal de oficina y $16 \%$ personal de servicio. Cabe señalar la importancia en esta composición de técnicos, obreros calificados y personal de oficina, pues refleja los procesos de reconversión industrial hacia un trabajo más calificado, de cuello blanco.

En este sentido, las empresas filiales y de grupo concentran mayores porcentajes de técnicos, supervisores y personal de oficina, mientras que las individuales y matrices, de obreros.

La séptima pregunta del cuestionario hace referencia al año de establecimiento de las empresas en Ciudad del Carmen y revela que lo hicieron entre 1920 y 2000. Sin embargo, puede observarse que los años clave fueron entre 1990 y 2000, cuando se asentó 48.84\% del total. La comparación entre los tipos de empresas muestra que las foráneas se instauraron con mayor frecuencia en los años sesenta, setenta y noventa (Cuadro 1). La baja en los años ochenta puede explicarse por la crisis petrolera ocasionada por una reducción en los costos internacionales, lo que hizo menos atractiva la región.

La octava pregunta consulta acerca de los productos y servicios prestados por las empresas entrevistadas; dentro de la amplia gama de bienes y servicios, las hay navieras, de servicios de posicionamiento por satélites, de servicios acuáticos, de mantenimiento, hotelería, construcción en general, sistemas de control industrial, estudios geofísicos y estudios geotécnicos, entre otros. 
Cuadro 1

Características generales (I) de las empresas: valor de las ventas, número de empleados, tipo de personal y año de establecimiento

\begin{tabular}{|c|c|c|c|}
\hline Características & $\begin{array}{l}\text { Total } \\
(\%)\end{array}$ & $\begin{array}{c}\text { Individuales } \\
\text { y matrices (\%) }\end{array}$ & $\begin{array}{c}\text { Filiales } y \\
\text { de grupo (\%) }\end{array}$ \\
\hline \multicolumn{4}{|l|}{$\begin{array}{l}\text { I. Valor de las ventas del último año } \\
\text { (en pesos de 2002) }\end{array}$} \\
\hline Menos de 500,000.00 & 2.33 & 0.00 & 7.14 \\
\hline Entre más de 500,000 y 1 millón & 11.63 & 17.24 & 0.00 \\
\hline Entre más de 1 y 3 millones & 16.28 & 13.79 & 21.43 \\
\hline Entre más de 3 y 5 millones & 4.65 & 6.90 & 0.00 \\
\hline Entre más de 5 y 8 millones & 13.95 & 10.34 & 21.43 \\
\hline Entre más de 8 y 12 millones & 4.65 & 6.90 & 0.00 \\
\hline Entre más de 12 y 15 millones & 4.65 & 0.00 & 14.29 \\
\hline Entre más de 15 y 20 millones & 4.65 & 6.90 & 0.00 \\
\hline Más de 20 millones & 37.21 & 37.93 & 35.71 \\
\hline Total & 100.00 & 100.00 & 100.00 \\
\hline \multicolumn{4}{|l|}{ II. Número de empleados } \\
\hline Hasta 30 & 58.15 & 48.28 & 78.57 \\
\hline De 31 a 60 & 13.95 & 13.79 & 21.43 \\
\hline De 61 a 100 & 13.95 & 17.24 & 0.00 \\
\hline De 101 a 400 & 9.30 & 13.79 & 0.00 \\
\hline De 401 a 1200 & 4.65 & 6.90 & 0.00 \\
\hline Total & 100.00 & 100.0 & 100.00 \\
\hline \multicolumn{4}{|l|}{ III. Tipo de personal } \\
\hline Directivos o gerentes & 13.70 & 14.76 & 11.50 \\
\hline Técnicos o supervisores & 14.25 & 10.81 & 21.45 \\
\hline Obreros calificados & 20.75 & 23.29 & 15.61 \\
\hline Obreros no calificados & 7.90 & 9.21 & 5.18 \\
\hline Personal de oficina & 27.40 & 24.61 & 33.34 \\
\hline Personal de servicio & 16.00 & 17.31 & 12.92 \\
\hline Total & 100.00 & 100.00 & 100.00 \\
\hline \multicolumn{4}{|l|}{ IV. Año de establecimiento } \\
\hline Entre 1920 y 1940 & 2.33 & 3.45 & 0.00 \\
\hline Entre 1941 y 1960 & 4.65 & 3.45 & 7.14 \\
\hline Entre 1961 y 1980 & 20.93 & 17.24 & 28.57 \\
\hline Entre 1981 y 1990 & 18.60 & 24.14 & 7.14 \\
\hline Entre 1991 y 2000 & 48.84 & 48.28 & 50.00 \\
\hline Entre 2001 y 2002 & 4.65 & 3.45 & 7.14 \\
\hline Total & 100.00 & 100.00 & 100.00 \\
\hline
\end{tabular}

Fuente: elaboración propia con base en la encuesta. 
Cuadro 2

Características generales (II) de las empresas: número de productos producidos (servicios prestados), tipos y número de insumos utilizados

\begin{tabular}{lrrr}
\hline \multicolumn{1}{c}{ Características } & $\begin{array}{c}\text { Total } \\
(\%)\end{array}$ & $\begin{array}{c}\text { Individuales } \\
\text { ymatrices }(\%)\end{array}$ & $\begin{array}{c}\text { Filiales } y \\
\text { de grupo (\%) }\end{array}$ \\
\hline I. Número de productos o servicios & & & \\
1 servicio & 51.16 & 55.18 & 42.86 \\
2 servicios & 20.93 & 17.24 & 28.57 \\
3 servicios & 13.95 & 10.34 & 21.43 \\
4 servicios & 6.98 & 6.90 & 7.14 \\
5 servicios & 6.98 & 10.34 & 0.00 \\
Total & 100.00 & 100.00 & 100.00 \\
& & & \\
II. Tipos de insumos utilizados & & & \\
Naturales & 16.28 & 6.76 & 3.93 \\
Industrializados & 83.72 & 93.24 & 96.07 \\
Total & 100.00 & 100.00 & 100.00 \\
& & & \\
III. Número de insumos utilizados & & & \\
1 insumo & 63.01 & 58.97 & 65.00 \\
2 insumos & 22.56 & 23.62 & 22.50 \\
3 insumos & 9.77 & 10.34 & 10.00 \\
4 insumos & 4.19 & 5.69 & 2.50 \\
insumos & 0.47 & 1.38 & 0.00 \\
Total & 100.00 & 100.00 & 100.00 \\
\hline
\end{tabular}

Fuente: elaboración propia con base en la encuesta.

El Cuadro 2 muestra que la mayoría de empresas (51.16\%) prestan un solo servicio o producto, y conforme aumenta su número, disminuye el de compañías que los ofrecen. El conjunto de las empresas individuales y matrices está más diversificado que el de las filiales o de grupo; más de $10 \%$ de las primeras ofrecen cinco o más servicios o productos, mientras que ninguna de las filiales y de grupo ofrece tal cantidad.

La novena pregunta se refiere a los tipos de insumos utilizados y el porcentaje que corresponde a los naturales (que no requieren ningún proceso de transformación) y a los industrializados. Del total, $83.72 \%$ requiere éstos, mientras que $16.28 \%$ utiliza los naturales (Cuadro 2). Como puede verse, las diferencias entre empresas de diverso tipo de propiedad no son tan importantes como en otros.

La décima pregunta intenta conocer los principales insumos utilizados y el porcentaje de costos que representan. Dentro de la amplia gama se encuentran combustibles (diesel marino), refacciones para maquinaria pesada, equipos de medición, acero, soldadura, arena sílice, pintura, equipos de seguridad, cementos y químicos especiales, entre otros. Se encontró que $63.02 \%$ utiliza un solo insumo, $22.56 \%$ utiliza dos, $9.77 \%$ tres, $4.19 \%$ 
Cuadro 3

Integración territorial de las empresas en cuanto a procedencia de trabajadores, origen de las compras y destino de las ventas

\begin{tabular}{|c|c|c|c|}
\hline Concepto & $\begin{array}{l}\text { Total } \\
(\%)\end{array}$ & $\begin{array}{c}\text { Individuales y } \\
\text { matrices }(\%)\end{array}$ & $\begin{array}{c}\text { Filiales } y \\
\text { de grupo }(\%)\end{array}$ \\
\hline \multicolumn{4}{|l|}{ I. Procedencia de los trabajadores } \\
\hline Local (municipio de Carmen) & 64.03 & 66.56 & 58.80 \\
\hline Regional (estado de Campeche y aledaños) & 12.23 & 11.66 & 13.41 \\
\hline De la ciudad de México & 2.36 & 3.33 & 0.37 \\
\hline País (resto de los estados) & 18.13 & 17.77 & 18.88 \\
\hline De Estados Unidos y Canadá & 2.56 & 0.69 & 6.43 \\
\hline De otros países & 0.69 & 0.00 & 2.12 \\
\hline Total & 100.00 & 100.00 & 100.00 \\
\hline \multicolumn{4}{|l|}{ II. Origen de las compras } \\
\hline Local (municipio de Carmen) & 34.29 & 36.71 & 29.29 \\
\hline Regional (estado de Campeche y aledaños) & 4.09 & 6.07 & 0.00 \\
\hline De la ciudad de México & 21.95 & 22.55 & 20.71 \\
\hline País (resto de los estados) & 25.83 & 24.16 & 29.29 \\
\hline De Estados Unidos y Canadá & 11.16 & 10.00 & 13.57 \\
\hline De otros países & 2.67 & 0.52 & 7.14 \\
\hline Total & 100.00 & 100.01 & 100.00 \\
\hline \multicolumn{4}{|l|}{ III. Destino de las ventas } \\
\hline Local (municipio de Carmen) & 77.02 & 76.45 & 78.21 \\
\hline Regional (estado de Campeche y aledaños) & 10.47 & 14.14 & 2.86 \\
\hline De la ciudad de México & 6.40 & 2.07 & 15.36 \\
\hline País (resto de los estados) & 5.07 & 5.79 & 3.57 \\
\hline De Estados Unidos y Canadá & 0.81 & 1.21 & 0.00 \\
\hline De otros países & 0.23 & 0.34 & 0.00 \\
\hline Total & 100.00 & 100.00 & 100.00 \\
\hline
\end{tabular}

Fuente: elaboración propia con base en la encuesta.

cuatro y $0.47 \%$ de negocios usa cinco insumos o más. El Cuadro 2 sugiere mayor uso en el número de insumos por parte de las empresas individuales y matrices.

Integración territorial en el empleo, las compras y las ventas

La pregunta 11 del instrumento investiga el origen territorial de sus trabajadores. Para el total de empresas de la muestra, las cifras obtenidas evidencian que $64.03 \%$ de los trabajadores procede de la propia localidad; $12.23 \%$ de la región (estados de Campeche y aledaños: Tabasco, Yucatán y Chiapas); $2.36 \%$, de la ciudad de México; $18.13 \%$, del resto de los estados del país; 2.56\%, de Estados Unidos y Canadá, y 0.69\%, de otras naciones (Cuadro 3). 
Una diferencia importante entre los grupos de empresas está en la procedencia de sus empleados; en las filiales y de grupo, $8.55 \%$ de los trabajadores eran procedentes de diferentes partes del mundo; la cifra para compañísas individuales y matrices es de sólo $0.69 \%$.

La pregunta 12 indaga la procedencia de las mercancías o materias primas que utilizan las empresas establecidas en Carmen. Aunque la mayor parte de los suministros se obtiene en la localidad (34.29\%), un alto porcentaje proviene de la ciudad de México (21.95\%), mientras que solamente $13.83 \%$ proviene del exterior (Cuadro 3). Aquí también, las empresas individuales y matrices están más integradas a la localidad que las filiales y de grupo, más integradas a áreas fuera de la región de Campeche, situación que resulta congruente con lo expuesto en la revisión de la literatura que señala la tendencia de las empresas filiales o de grupo por generar un menor flujo económico local y regional.

La pregunta 13 consulta acerca del destino de las ventas, del cual se encontró un grado muy alto de integración local de las empresas (77.02\% del total se realiza en la región), y una muy baja con áreas fuera del municipio de Carmen (Cuadro 3).

Una diferencia importante entre grupos de empresas está en la integración regional de las ventas; mientras que las empresas individuales y matrices venden más de $90 \%$ de sus productos o servicios en los mercados locales o regionales de Campeche, las filiales y de grupo venden sólo alrededor de $81 \%$. Por otro lado, las filiales y de grupo venden $15.36 \%$ del total en la ciudad de México, mientras que las individuales y matrices comercializan allí sólo $2.07 \%$.

\section{Los factores de localización}

En los cuadros siguientes se presenta un resumen de los resultados obtenidos en cuanto a la importancia que algunos factores tuvieron en la decisión de localización de las empresas incluidas en la muestra. Primero, se expone la importancia que éstas dieron a cada uno de los 17 grupos de factores de localización, mostrándose estos resultados para el total de las compañías, para las individuales y matrices, y para las filiales y de grupo. Posteriormente, se señala la relevancia que dentro de cada grupo dieron a los factores de manera individual. Estos resultados también se presentan para el total de las empresas y para los grupos ya mencionados.

En el Cuadro 4 se puede observar que los tres grupos de factores más importantes para el global de la muestra son el mercado, la calidad de vida y la mano de obra; fueron menos relevantes la competencia, el gobierno y el agua.

Para el caso de las empresas individuales y matrices, los grupos más y menos importantes son muy semejantes al total, excepto por los factores personales, que para las individuales y matrices figuran en tercer lugar, sustituyendo a la mano de obra.

Para las empresas filiales y de grupo, los factores más importantes siguen siendo mercado y calidad de vida pero, en este caso, aparecen en segundo lugar transporte y residuos.

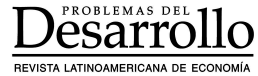


JORGE A. DURÁN E. Y MARIO M. CARRILLO H.

Cuadro 4

Importancia de los grupos de factores de localización

\begin{tabular}{lccc}
\hline Grupo de factores & Total & $\begin{array}{c}\text { Individuales } y \\
\text { matrices }\end{array}$ & $\begin{array}{c}\text { Filiales } y \\
\text { de grupo }\end{array}$ \\
\hline Mercado & 3.96 & 4.03 & 3.81 \\
Calidad de vida & 3.24 & 3.23 & 3.26 \\
Mano de obra & 3.15 & 3.11 & 3.23 \\
Personales & 3.13 & 3.18 & 3.02 \\
Infraestructura & 3.09 & 3.09 & 3.11 \\
Insumos & 3.07 & 3.16 & 2.89 \\
Interacción & 3.06 & 3.14 & 2.91 \\
Clima & 3.06 & 3.16 & 2.86 \\
Proveedores & 3.01 & 2.97 & 3.07 \\
Servicios financieros & 3.00 & 3.08 & 2.83 \\
Residuos & 2.98 & 2.79 & 3.38 \\
Transporte & 2.96 & 2.86 & 3.38 \\
R\&D & 2.88 & 2.77 & 3.12 \\
Energía & 2.81 & 2.77 & 3.12 \\
Agua & 2.75 & 2.67 & 2.91 \\
Gobierno & 2.70 & 2.52 & 3.09 \\
Competencia & 2.69 & 2.53 & 3.00 \\
\hline
\end{tabular}

Fuente: elaboración propia con base en la encuesta.

Este último se refiere a las facilidades con las cuales se cuenta en la localidad para el tratamiento y disposición final de los residuos industriales. Los menos importantes para este grupo de empresas son los servicios financieros, el clima y los insumos.

En el Cuadro 5, columnas $a$ y $b$, se muestran los puntajes que obtuvieron los factores individuales dentro de cada grupo para el total de las empresas, indicando en orden descendente su importancia. En las columnas $c$ y $d$, se muestran esos puntajes para el caso del grupo de empresas individuales o matrices; en las columnas $e$ y $f$ se registran los resultados correspondientes a las empresas filiales y de grupo.

Así, se puede observar para el total de empresas en el Cuadro 5 que, en relación con la importancia que recibe el mercado, destaca el tamaño, la cercanía, el contacto directo con el consumidor y la capacidad de absorción del mercado local. En cuanto a la calidad de vida de la zona, se pone énfasis en el entorno físico agradable. En relación con la mano de obra, se indican como los factores más importantes la cantidad y la disponibilidad en la localidad.

Como se puede ver de las columnas $c$ y $d$ del Cuadro 5, los factores que las empresas individuales y matrices consideran muy relevantes son parecidos a los mencionados por el total. Sin embargo, en este caso destacan, dentro de los factores personales, la cercanía a la familia y las recomendaciones de terceros para establecer sus negocios en la localidad.

Finalmente, en relación con las empresas filiales y de grupo, y con referencia al grupo de factores relacionados con residuos, destacan las facilidades que encuentran en la localidad para su disposición final, así como poder cumplir fácilmente con las leyes aplicables y los costos de la eliminación de los residuos (columnas $e$ y $f$ del Cuadro 5).

\section{DeSarrollo}


Cuadro 5

Importancia de los factores de localización al nivel individual para las empresas

\begin{tabular}{|c|c|c|c|c|c|c|c|}
\hline \multirow[b]{2}{*}{$\begin{array}{l}\text { Grupo de } \\
\text { factores }\end{array}$} & \multirow[b]{2}{*}{ Factor } & \multicolumn{2}{|c|}{ Totales } & \multicolumn{2}{|c|}{$\begin{array}{l}\text { Individuales y } \\
\text { matrices }\end{array}$} & \multicolumn{2}{|c|}{$\begin{array}{l}\text { Filiales y de } \\
\quad \text { grupo }\end{array}$} \\
\hline & & $\begin{array}{l}\text { Prom. } \\
\text { (a) }\end{array}$ & $\begin{array}{l}\text { Prom./ } \\
\text { Prom. } \\
\text { (b) }\end{array}$ & $\begin{array}{l}\text { Prom. } \\
\text { (c) }\end{array}$ & $\begin{array}{l}\text { Prom./ } \\
\text { Prom. } \\
\text { (d) }\end{array}$ & $\begin{array}{l}I \\
\text { Prom. } I \\
\text { (e) }\end{array}$ & $\begin{array}{l}\text { Prom/ } \\
\text { Prom. } \\
\quad(f)\end{array}$ \\
\hline \multirow[t]{5}{*}{ Mercado } & 29. Tamaño del mercado & 4.28 & & 4.34 & & 4.14 & \\
\hline & 26. Cercanía al cliente & 4.23 & & 4.24 & & 4.21 & \\
\hline & 28. Contacto directo con el consumidor & 4.21 & & 4.41 & & 3.79 & \\
\hline & 27. Capacidad de absorción del mercado local & 4.16 & & 4.34 & & 3.79 & \\
\hline & 30. Establecimiento en una zona altamente poblada & 2.93 & 3.96 & 2.83 & 4.03 & 3.14 & 3.81 \\
\hline \multirow{3}{*}{$\begin{array}{l}\text { Calidad } \\
\text { de vida }\end{array}$} & 53. Calidad de vida en la zona & 3.42 & & 3.38 & & 3.50 & \\
\hline & 54. Entorno físico agradable & 3.42 & & 3.52 & & 3.21 & \\
\hline & 55. Centros de esparcimiento y cultura & 2.88 & 3.24 & 2.79 & 3.23 & 3.07 & 3.26 \\
\hline \multirow{5}{*}{$\begin{array}{l}\text { Mano } \\
\text { de obra }\end{array}$} & 14. Cantidad disponible & 3.37 & & 3.41 & & 3.29 & \\
\hline & 15. Disponibilidad de mano de obra calificada & 3.37 & & 3.31 & & 3.50 & \\
\hline & 16. Nivel de salarios en la región & 3.30 & & 3.31 & & 3.31 & \\
\hline & 17. Actitudes de la mano de obra & 3.00 & & 2.97 & & 3.07 & \\
\hline & 18. Actividad sindical o relaciones obrero-patronales & 2.70 & 3.15 & 2.55 & 3.11 & 3.00 & 3.23 \\
\hline \multirow{4}{*}{$\begin{array}{l}\text { Perso- } \\
\text { nales }\end{array}$} & 51. Cercanía a la familia & 3.35 & & 3.41 & & 3.21 & \\
\hline & 52. Recomendaciones y sugerencias & & & & & & \\
\hline & de terceros para establecerse en la zona & 3.09 & & 3.21 & & 2.86 & \\
\hline & 50. Atracción personal al momento de elegir la zona & 2.95 & 3.13 & 2.93 & 3.18 & 3.00 & 3.02 \\
\hline \multirow{4}{*}{$\begin{array}{l}\text { Infraes- } \\
\text { tructura }\end{array}$} & 36. Costo del terreno & 3.35 & & 3.41 & & 3.21 & \\
\hline & 38. Infraestructura disponible en el terreno & 3.12 & & 3.17 & & 3.00 & \\
\hline & 37. Disponibilidad de terrenos para futuras expansiones & 3.07 & & 3.07 & & 3.07 & \\
\hline & 39. Financiamientos para la adquisición de terrenos & s 2.84 & 3.09 & 2.69 & 3.09 & 3.14 & 3.11 \\
\hline \multirow[t]{4}{*}{ Insumos } & 2. Disponibilidad & 3.19 & & 3.21 & & 3.14 & \\
\hline & 1. Calidad & 3.16 & & 3.21 & & 3.07 & \\
\hline & 4. Costo & 2.98 & & 3.03 & & 2.86 & \\
\hline & 3. Cercanía & 2.95 & 3.07 & 3.17 & 3.16 & 2.50 & 2.89 \\
\hline \multirow{4}{*}{$\begin{array}{l}\text { Inter- } \\
\text { acción }\end{array}$} & 43. Relación con empresas & 3.58 & & 3.76 & & 3.21 & \\
\hline & 42. Disponibilidad de servicios en la zona & 3.21 & & 3.28 & & 3.07 & \\
\hline & 44. Interacción con otras empresas & 2.88 & & 2.90 & & 2.86 & \\
\hline & $\begin{array}{l}\text { 41. Posibilidad de compartir recursos } \\
\text { e instalaciones con otras empresas }\end{array}$ & 2.58 & 3.06 & 2.62 & 3.14 & 2.50 & 2.91 \\
\hline \multirow[t]{2}{*}{ Clima } & 57. Certidumbre de aspectos climáticos & 3.09 & & 3.17 & & 2.93 & \\
\hline & 56. Clima de la región & 3.02 & 3.06 & 3.14 & 3.16 & 2.79 & 2.86 \\
\hline
\end{tabular}


Cuadro 5 (continuación)

\begin{tabular}{|c|c|c|c|c|c|c|c|}
\hline & & Totc & ales & $\begin{array}{l}\text { Individu } \\
\text { matr }\end{array}$ & $\begin{array}{l}\text { uales } y \\
\text { ices }\end{array}$ & $\begin{array}{r}\text { Filiale } \\
\text { gru }\end{array}$ & $\begin{array}{l}\text { es y de } \\
\text { upo }\end{array}$ \\
\hline $\begin{array}{l}\text { Grupo de } \\
\text { factores }\end{array}$ & Factor & $\begin{array}{l}\text { Prom. } \\
\text { (a) }\end{array}$ & $\begin{array}{l}\text { Prom./ } \\
\text { Prom. } \\
\text { (b) }\end{array}$ & $\begin{array}{l}\text { Prom. } \\
\text { (c) }\end{array}$ & $\begin{array}{l}\text { Prom./ } \\
\text { Prom. } \\
\text { (d) }\end{array}$ & $\begin{array}{l}\text { Prom. } \\
(e)\end{array}$ & $\begin{array}{c}\text { Prom/ } \\
\text { Prom. } \\
\quad(f)\end{array}$ \\
\hline $\begin{array}{l}\text { Provee- } \\
\text { dores }\end{array}$ & 7. Calidad & 3.28 & & 3.17 & & 3.50 & \\
\hline & 5. Relación con los proveedores & 3.07 & & 3.21 & & 2.79 & \\
\hline & 8. Disponibilidad & 2.95 & & 2.79 & & 3.29 & \\
\hline & 6. Cercanía & 2.72 & 3.01 & 2.72 & 2.97 & 2.71 & 3.07 \\
\hline Servicios & 31. Cercanía a las instituciones de crédito & 3.05 & & 3.07 & & 3.00 & \\
\hline & 33. Costo del capital & 3.02 & & 3.07 & & 2.93 & \\
\hline & $\begin{array}{l}\text { 32. Facilidad de otorgamiento de servicios } \\
\text { por parte de las instituciones de crédito }\end{array}$ & 2.93 & 3.00 & 3.10 & 3.08 & 2.57 & 2.83 \\
\hline Residuos & 34. Facilidad en su desecho & 3.09 & & 2.93 & & 3.43 & \\
\hline & $\begin{array}{l}\text { 35. Facilidad de cumplimiento de leyes o } \\
\text { regulaciones en su eliminación }\end{array}$ & 3.05 & & 2.86 & & 3.43 & \\
\hline & 40. Costo de su eliminación & 2.81 & 2.98 & 2.59 & 2.79 & 3.29 & 3.38 \\
\hline Trans- & 9. Calidad de los medios de transporte & 3.14 & & 3.07 & & 3.29 & \\
\hline porte & 10. Disponibilidad y variedad & 2.98 & & 2.93 & & 3.07 & \\
\hline & 12. Costo & 2.93 & & 2.72 & & 3.36 & \\
\hline & 13. Seguridad & 2.93 & & 2.83 & & 3.14 & \\
\hline & 11. Acceso y cercanía & 2.84 & 2.96 & 2.72 & 2.86 & 3.07 & 3.19 \\
\hline$R \& D$ & 47. Acceso a tecnología & 3.21 & & 3.21 & & 3.21 & \\
\hline & 46. Existencia de bibliotecas, escuelas & & & & & & \\
\hline & y universidades en la zona & 2.98 & & 2.86 & & 3.21 & \\
\hline & 45. Cercanía a compañías dedicadas a la investigación & 2.47 & 2.88 & 2.24 & 2.77 & 2.93 & 3.12 \\
\hline Energía & 19. Costo & 3.23 & & 3.24 & & 3.21 & \\
\hline & 21. Confiabilidad en los servicios & 3.05 & & 3.00 & & 3.14 & \\
\hline & 20. Instalaciones no comunes & 2.16 & 2.81 & 2.07 & 2.77 & 2.36 & 2.90 \\
\hline Agua & 22. Disponibilidad & 3.16 & & 3.17 & & 3.14 & \\
\hline & 25. Confiabilidad en el servicio & 2.74 & & 2.59 & & 3.07 & \\
\hline & 24. Costo del agua & 2.60 & & 2.48 & & 2.86 & \\
\hline & 23. Características específicas & 2.49 & 2.75 & 2.45 & 2.67 & 2.57 & 2.91 \\
\hline Gobierno & 58. Simplificación de trámites y o permisos & 3.14 & & 3.07 & & 3.29 & \\
\hline & 59. Incentivos gubernamentales & 2.84 & & 2.66 & & 3.21 & \\
\hline & 60. Asesorías gubernamentales & 2.49 & & 2.17 & & 3.14 & \\
\hline & $\begin{array}{l}\text { 61. Programas de capacitación ofrecidos } \\
\text { por el gobierno }\end{array}$ & 2.35 & 2.70 & 2.17 & 2.52 & 2.71 & 3.09 \\
\hline $\begin{array}{l}\text { Compe- } \\
\text { tencia }\end{array}$ & 48. Cercanía & 3.07 & & 3.07 & & 3.07 & \\
\hline & 49. Existencia de uniones & 2.30 & 2.69 & 2.00 & 2.53 & 2.93 & 3.00 \\
\hline
\end{tabular}

Fuente: elaboración propia con base en la encuesta. 


\section{Conclusiones}

El objetivo principal de este trabajo ha sido presentar los resultados de un estudio realizado en 2002 sobre relaciones interregionales y factores de localización, con el objeto de identificar una cartera de proyectos regionales de inversión que ayudara a compensar las posibles afectaciones económicas de un proyecto de explotación de gas en la península de Atasta y en Ciudad del Carmen, Campeche.

Para ello, se trató de: $a$ ) identificar (mediante la aplicación de una encuesta a 58 empresas industriales) actividades económicas que, habiendo resultado las de mayor valor presente neto, tuvieran los mayores grados de integración regional; y $b$ ) reconocer los factores que más habían influido en la decisión de localización de las empresas de la región, para efectos de promover la instalación de compañías nuevas en las ramas seleccionadas.

Para lograr ese objetivo, se revisaron algunos aspectos teóricos y prácticos de los factores de localización económica. Se anotó la manera en que se ha puesto énfasis en las economías externas como factor de atracción para las empresas al momento de localizarse, llegando a concretarse en un extremo en los clusters industriales. También se revisó la importancia atribuida en México a aspectos de infraestructura, políticas gubernamentales o, bien, a iniciativa de las empresas por encontrar asociaciones estratégicas, ya sea de tipo vertical u horizontal.

Sin embargo, dada las características de Ciudad del Carmen, fue necesario consultar experiencias y estudios sobre factores de localización en las llamadas regiones periféricas o no metropolitanas. En particular, se observó que el impacto que pueden tener éstos en las empresas se encuentra diferenciado por su grado de localidad. Al respecto, se plantea una distinción entre los factores de localización y su relación con las empresas locales (individuales y matrices) versus las foráneas (filiales y de grupo), tanto nacionales como multinacionales. ${ }^{6}$

Los resultados más relevantes de la encuesta aplicada entre las empresas que prestan servicio a Petróleos Mexicanos son los siguientes:

a) No hay diferencias significativas en cuanto al tamaño entre las empresas foráneas y locales en términos del volumen de ventas, aunque las primeras tienden a ocupar menos personal.

b) Las empresas foráneas han sido ligeramente más activas (que las locales) en establecerse en la localidad en los últimos diez años.

6 Según la experiencia de dos países, Noruega y Gran Bretaña, es posible que mediante diversas intervencionesorganizadas, de autoridades municipales, estatales o federales, o deasociaciones empresariales, se propicie un mayor flujo económico de las empresas en la localidad, ya sea mediante la creación de instituciones educativas, promotoras, o bien de empresas vinculadas con las actividades industriales motrices, como astilleros y centros de reparación de maquinaria de perforación, entre otros.

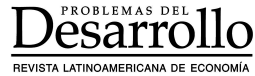


c) Las empresas foráneas tienden a ofrecer de dos a tres líneas de productos, y se especializan más que las locales.

d) En general, todas las empresas tienen bajo porcentaje en el uso de insumos naturales; la gran parte de adquisiciones requieren algún proceso de industrialización.

e) El porcentaje de mano de obra local para el total de empresas es de $64.03 \%$ y resulta menor para las foráneas (menos de 58.80\%). Las necesidades de éstas se enfocan más a personal técnico y de oficina, en tanto que las locales contratan más directivos, obreros calificados y de servicio.

f) El porcentaje de compras que se realiza en la localidad es bajo para el total de las empresas, pero lo es más para las foráneas, menor a $30 \%$. Sus adquisiciones se centran fundamentalmente en la ciudad de México y el resto del país.

En lo correspondiente a los factores de localización a los cuales dieron mayor importancia las empresas cuando se establecieron, se tiene que:

a) Para el total de las empresas, el mercado fue el factor principal para decidir su ubicación, sobre todo la cercanía y tamaño de ventas potenciales que representa PEMEX.

b) La calidad de vida figuró también como un factor de importancia para las compañías.

c) En cuanto a la infraestructura, ésta ocupa una posición de importancia intermedia para el conjunto de las empresas, aunque para las foráneas es importante encontrar instalaciones que faciliten la disposición de sus residuos industriales.

d) Para el total de las empresas resultaron poco importantes los insumos locales, la competencia y las facilidades que pudieran otorgar el gobierno.

e) El transporte resultó un factor de mediana importancia para el total de las empresas, y mayor para las foráneas.

f) La interacción con empresas locales es, en general, de mediana importancia, siendo un factor todavía de menor peso para las foráneas.

En resumen, este estudio permite verificar los resultados obtenidos en otros países y, a su vez, proporciona una visión más precisa para la implantación de las acciones que pretende llevar a cabo la RMNE en la localidad.

De hecho, los resultados del estudio proporcionaron orientaciones importantes para definir la cartera de proyectos que posteriormente fue desarrollada, en especial la relevancia del mercado local en el establecimiento de empresas en la región. Así, se juzgó conveniente generar propuestas en las cuales participaran, de manera preponderante, la población originaria de la región, en la propiedad y en la dirección de los proyectos. Los beneficios que, en términos de captación de mano de obra y de compra de insumos que caracterizan a las empresas locales, apoyan esta decisión, además de que este tipo de compañías tienden 
a una menor sujeción a las variaciones cíclicas de la industria petrolera. El análisis de los factores también proporcionó información valiosa para determinar de qué manera reforzar o consolidar el apoyo a los proyectos incluidos en la cartera.

\section{Bibliografía}

Barkley, David L. y Sylvain Hinschberger, "Industrial Restructuring: Implications for the Decentralization of Manufacturing to Nonmetropolitan Areas", en Economic Development Quaterly, núm. 6, 1992, pp. 64-79.

Bellak, C. y J. Cantwell, "Globalization Tendencies Relevant for Latecomers", en M. Storper; S.B. Thormadikis y L.J. Tsipouri (editores), Latecomers en the Global Economy, Londres, Routledge, 1998, pp. 40-75.

Bennett, Robert J.; Paul J.A. Robson y William J.A. Bratton, "The Influence of LOcation on the Use by sMEs of External Advice y Collaboration", en Urban Studies, vol. 38, núm. 9, 2001,pp. 1531-1557.

Boomsma, P.E. y J. Oosterhaven, "A Double Entry Method for the Construction of Regional Inputoutput Tables", en Journal of Regional Sciences, vol. 32, 1992, pp. 265-284.

Buetre, L. Benjamin y Fredoun Ahmadi-Esfahani, "Updating an Input-Output Table for Use in Policy Analysis", en The Australian Journal of Agricultural and Resource Economics, vol. 44, núm. 4, 2002, pp. 573-603.

Carrillo H. y M. Mario, Aspectos microeconómicos introductorios del desarrollo regional y urbano, México, UPIICSA-IPN, 2002.

Chapman, Keith y David Walter, Industrial Location, Principles and Policies, Londres, Basil Blackwell, 1987.

Christerson, B. y C. Lever-Tracy, "The Third China? Emerging Industrial Districts in rural China", en International Journal of Urban and Regional Research, núm. 21, 1997, pp. 569-588.

Cumbers, Andrew, "Globalization, Local Economic Development and the Branch Plant Region: The case of the Aberdeen Oil Complex", en Regional Studies, vol. 34, núm. 4, 2000a, pp. 371-382.

Cumbers, Andrew, "The National State as Mediator of Regional Development Outcomes in a Global Era", en European Urban and Regional Studies, vol. 7, núm. 3, 2000b, pp. 237-252.

Ellison, Glenn y Edward L. Glaeser, "Geographic Concentration in U.S. Manufacturing Industries: A Dartboard Approach", en Journal of Political Economy, núm. 105, 1997, pp. 889-927.
Enright, Michael J., "The Determinants of Geographic Concentration in Industry", en Working Paper, núm. 93-052, Division of Research, Harvard Business School, 1993.

__- "Organization and Coordination in Geographically Concentrated Industries", en D. Raff y N.R. Lamoraux (editores), Coordination and Information: Historical Perspectives on the Organization of Enterprise, Chicago, Chicago University Press for NBER, 1994, pp. 103-142.

Garza, Gustavo, Desconcentración, tecnología y localización industrial en México, México, El Colegio de México, 1999.

Gereffi, G., "Global Production Systems and Third World Development", en B. Stallings (editor), Global Change, Regional Response: The New International Context of Development, Cambridge, Cambridge University Press, 1995, pp. 100-142.

Glasson, John, An Introduction to Regional Planning, Londres, Hutchinson, 1980.

Graizbord, Boris, "Estructura y posibilidades de crecimiento de 22 ciudades industriales mexicanas", en Comercio Exterior, México, febrero de 1993.

Henderson, J. Vernon, "Efficiency and Resource Usage and City Size", en Journal of Urban Economics, núm. 19, 1986, pp. 47-70.

- "Externalities and Industrial Development", en Journal of Urban Economics, núm. 42, 1997, pp. 449-470.

Hernández Laos, Enrique, “The Sources of Regional Differences in Efficiency. The Case of Mexican Manufacturing", tesis de doctorado, East Anglia, University of East Anglia, 1977.

Herzog, Henry W. y Alan M. Schlottman (editores), Industrial Location and Public Policy, Knoxville, University of Tennessee Press, 1991.

Hewings, G.J.A. y R. Jonem, "Regional, Interregional and Multiregional Input-output Analysis", en P. Nijtomi (editor), Handbook of Regional and Urban Economics, Amsterdam, North-Holland, 1986, pp. 295-355.

Holmes, Thomas J., "Localization of Industry and Vertical Disintegration", en Staff Report 190, Federal Reserve Bank of Minneapolis, Minnesota, abril de 1995.

\section{$\underline{\text { DeSarrolollo }}$}


Isard, Walter, Métodos de análisis regional, Barcelona, Ariel, 1971.

Kim, Yunsoo; David L. Barkley y Mark S. Henry, "Industry Characteristics Linked to Establishment Concentrations in Nonmetropolitan Areas", en Journal of Regional Science, vol. 40, núm. 2, 2000, pp. 231-259.

Kloosterman, Robert C., "Clustering of Economic Activities in Policentric Urban Regions: The Case of the Randstad", en Urban Studies, vol. 38, núm. 4, 2001, pp. 717-732.

Krugman, Paul, Geography and Trade, Leuven, Leuven University Press, 1991.

Leontieff, W., Input-Output Economics, Nueva York, Oxford University Press, 1966.

Malecki, Edward J., Technology and Economic Development, Nueva York, John Wiley \& Sons, 1991.

Marshall, A., Principios de economía, 8a. ed., Londres, MacMillan, 1920.

Meyer-Stamer, Jörg, "Estrategias de desarrollo territorial basadas en el concepto de competitividad sistémica”, en Mercado de Valores, México, septiembre, 2000a, pp. 48-60.

, "Estrategias de desarrollo local y regional: clusters, política de localización y competitividad sistémica”, en Mercado de Valores, México, septiembre, 2000b, pp. 18-31

Peneder, Michael, Entrepreneurial Competition and Industrial Location, Cheltenham, Northampton, Edward Elgar, 2001.
Porter, M.E., "Location, Competition, and Economic Development: Local Clusters in a Global Economy", en Economic Development Quarterly, vol. 14, febrero, 2000, pp. 15-35.

Richardson, H.W., Teoría del crecimiento regional, Madrid, Pirámide, 1977.

Roper, S.; J.H. Love; B. Ashcroft y S. Dunlop, "Industry and Location Effects on UK Plants Innovation Propensity", en The Annals of Regional Science, núm. 34, 2000, pp. 489-502.

Ruiz Durán, Clemente, "Mejores prácticas del desarrollo industrial local", en Mercado de Valores, México, octubre de 2000, pp. 26-34.

Scott, Allen, "Industrial Organization and Location: Division of Labor, The Firm, and Spatial Process", en Economic Geography, núm. 62, 1986, pp. 215-231.

, Regions and the World Economy; The Coming Shape of Global Production, Competition, and Political Order, Oxford, Oxford University Press, 1998.

Smith, Stephen M. y David L. Barkley, "Local Input Linkages of Rural High Technology Manufacturers", en Land Economics, núm.67, 1991, pp. 472-483.

Venables, Anthony J., "Economic Integration and Industrial Agglomeration", en The Economic and Social Review, núm. 26, 1994, pp. 1-7. 